\title{
Collapsing Focal Segmental Glomerulosclerosis Resulting from a Single Dose of Zoledronate
}

\author{
Javier A. Neyra ${ }^{a}$ Omkar U. Vaidya ${ }^{a} \quad$ Allen Hendricks ${ }^{b}$ \\ Kamalanathan K. Sambandam ${ }^{a}$ \\ ${ }^{a}$ Division of Nephrology, Department of Internal Medicine, and ${ }^{b}$ Department of Pathology, \\ University of Texas Southwestern Medical Center, Dallas, Tex., USA
}

\section{Key Words}

Focal segmental glomerulosclerosis · Bisphosphonates · Zoledronate

\begin{abstract}
Bisphosphonates are commonly used for the treatment of osteoporosis, Paget's disease, multiple myeloma and hypercalcemia. Collapsing focal segmental glomerulosclerosis (FSGS) is known to occur uncommonly with exposure to bisphosphonates, specifically pamidronate and alendronate; it has rarely and equivocally been reported with zoledronate therapy. We describe the case of a 36-year-old African American female with metastatic breast cancer who presented with nephrotic-range proteinuria and acute kidney injury within 2 weeks of exposure to a single dose of zoledronate. The patient had a partial recovery of her renal function and showed improved proteinuria to a subnephrotic level after discontinuing zoledronate. In contrast to 2 prior reports of zoledronate-induced collapsing FSGS, the causative role of the exposure described here is certain. Our case necessitates the addition of zoledronate to the list of known causes of collapsing FSGS. Furthermore, it highlights the importance of periodically monitoring renal function and urine protein excretion with the use of zoledronate, which allows prompt diagnosis and withdrawal of the drug to increase the probability of renal recovery.

(C) 2014 S. Karger AG, Basel
\end{abstract}

\section{Introduction}

Bisphosphonates are frequently used for the treatment of osteoporosis, Paget's disease, multiple myeloma and hypercalcemia [1-3]. These agents reduce bone turnover by inhibiting osteoclast activity $[4,5]$. Bisphosphonate therapy has been linked to cases of acute kidney 
Neyra et al.: Collapsing Focal Segmental Glomerulosclerosis Resulting from a Single Dose of Zoledronate

injury (AKI) [6-8], and some bisphosphonates have been associated with the occurrence of collapsing focal segmental glomerulosclerosis (FSGS) $[9,10]$. Pamidronate is the one most commonly associated with this glomerular disease $[9,11]$. To date, there exist only 2 cases in the literature which propose zoledronate as the trigger for the onset of collapsing FSGS [10, 12]. In both of these cases, the causal relationship between zoledronate exposure and collapsing FSGS is not clear. The time course was such that the development of collapsing FSGS may have been a primary event occurring by chance during the bisphosphonate course. Alternatively, the glomerulopathy may have been prompted by other drugs which the patients received and which are also known to cause this lesion. In contrast, we report a case of collapsing FSGS occurring within 2 weeks of a single dose of zoledronate. This was followed by partial renal recovery after discontinuing the medication and without immunosuppressive treatment. The clinical course described undoubtedly proves that zoledronate is the causative agent.

\section{Case Report}

A 36-year-old African American female with a history of asthma, hypertension, pulmonary embolism and metastatic breast cancer presented with fever 1 day after the administration of a single intravenous 4-mg dose of zoledronate. She received vancomycin and piperacillin/ tazobactam for 4 days and was discharged without antibiotics after she defervesced and showed negative culture results. She presented again 6 days later with anasarca and nonoliguric AKI. Her home medications consisted of gabapentin, promethazine, ranitidine, amlodipine, enoxaparin subcutaneously, albuterol inhaler and morphine. Her vital signs were a temperature of $36.6^{\circ} \mathrm{C}$, a blood pressure of $141 / 91 \mathrm{~mm} \mathrm{Hg}$, a pulse of 102 beats/min and an oxygen saturation of $97 \%$ breathing room air. The physical examination was remarkable for anasarca, distended neck veins and decreased breath sounds throughout her right hemithorax. The laboratory data displayed mild leukocytosis, thrombocytosis, hemoglobin $8.8 \mathrm{~g} /$ $\mathrm{dl}$ and serum creatinine $556.9 \mu \mathrm{mol} / \mathrm{l}(6.3 \mathrm{mg} / \mathrm{dl})$ [baseline $79.6 \mu \mathrm{mol} / \mathrm{l}(0.9 \mathrm{mg} / \mathrm{dl})]$. The urinalysis exhibited many granular casts and few white and red blood cell casts. A 24-hour urine collection revealed proteinuria of $3.5 \mathrm{~g} / 24 \mathrm{~h}$ without a paraprotein. The complete serological workup was negative; this included HIV, parvovirus, cytomegalovirus, hepatitis B and hepatitis C serologies, rapid plasma reagin, antineutrophil cytoplasmic antibodies, antiglomerular basement membrane antibodies and normal serum complements. Renal ultrasound showed normal-sized kidneys and no hydronephrosis. Given our patient's overall good functional status despite her metastatic cancer, a kidney biopsy was performed 16 days after the exposure to zoledronate. Light microscopy of the biopsy specimen exhibited a total of 27 glomeruli, 2 of which were segmentally to globally collapsed with overlying hyperplasia and hypertrophy of the parietal epithelial cells, and microcystic dilation of the tubules. Additionally, there was evidence of mild interstitial infiltrate and moderate acute tubular injury (fig. 1). Electron microscopy revealed podocyte foot process effacement without immune complex deposits (fig. 2).

The patient's renal disease course (serum creatinine and proteinuria) is described in figure 3. She remained nonoliguric and did not require renal replacement therapy. Her serum creatinine peaked at $556.9 \mu \mathrm{mol} / \mathrm{l}(6.3 \mathrm{mg} / \mathrm{dl})$ and proteinuria at $3.5 \mathrm{~g} / 24 \mathrm{~h}(350 \mathrm{mg} / \mathrm{mmol})$. After kidney biopsy results became available, the patient was started on low-dose lisinopril ( $2.5 \mathrm{mg}$ daily) and no additional zoledronate therapy was given. Approximately 6 weeks after zoledronate exposure, her proteinuria decreased to $90.5 \mathrm{mg} / \mathrm{mmol}\left(0.8 \mathrm{~g} / \mathrm{g}-1.73 \mathrm{~m}^{2}\right)$ by urine protein-to-creatinine ratio, her serum albumin improved from 3.1 to $4.1 \mathrm{~g} / \mathrm{dl}$, and her serum creatinine improved to $141.4 \mu \mathrm{mol} / \mathrm{l}(1.60 \mathrm{mg} / \mathrm{dl})$. 

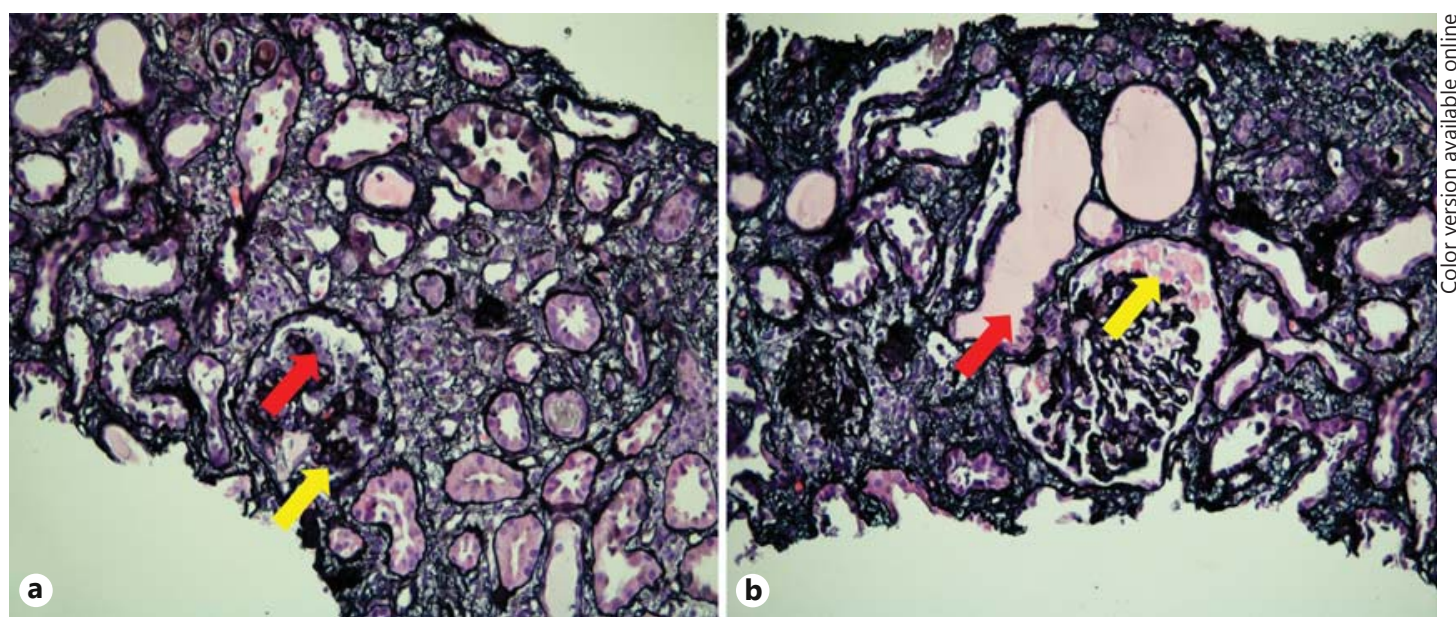

Fig. 1. Evaluation of the kidney biopsy specimen by light microscopy. a Glomerulus with global collapse of the glomerular tuft (yellow arrow) and podocyte hyperplasia (red arrow). Jones methenamine silver stain. $\times 200$. b Segmentally collapsed glomerulus with prominent protein droplets in visceral epithelial cells (yellow arrow) and acute tubular injury with microcystic dilation of some tubules (red arrow). Jones methenamine silver stain. $\times 200$. Colors refer to the online version only.

Fig. 2. Evaluation of the kidney biopsy specimen by electron microscopy showed capillary loop with prominent foot process effacement (arrow). Electron photomicrograph. $\times 4,000$.

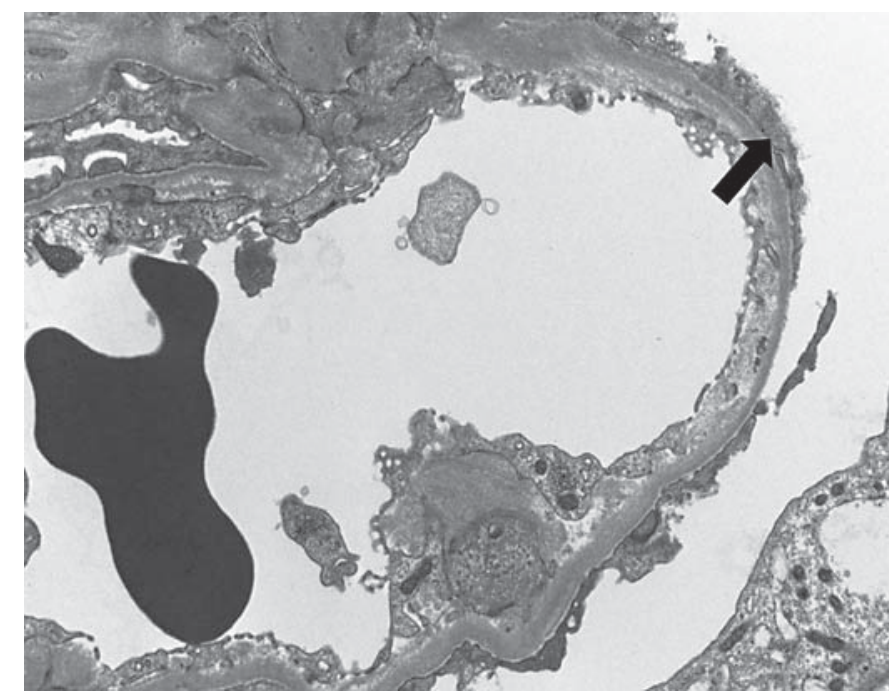

To expand on her cancer history, the patient was diagnosed with right breast carcinoma (estrogen/progesterone positive, human epidermal growth factor receptor 2 negative) about 8 years before the aforementioned presentation. She was treated with modified mastectomy and neoadjuvant chemotherapy (doxorubicin and cyclophosphamide) in addition to tamoxifen. The patient was then lost to follow-up until she presented 4 years later with metastases and was started on bevacizumab and paclitaxel. Four cycles were administered prior to discontinuing this regimen due to the development of proteinuria. Her proteinuria eventually improved to only trace on semiquantitative dipstick urinalysis. She was then treated with gemcitabine, vinorelbine tartrate and paclitaxel until her most recent presentation described above. 
Neyra et al.: Collapsing Focal Segmental Glomerulosclerosis Resulting from a Single Dose of Zoledronate

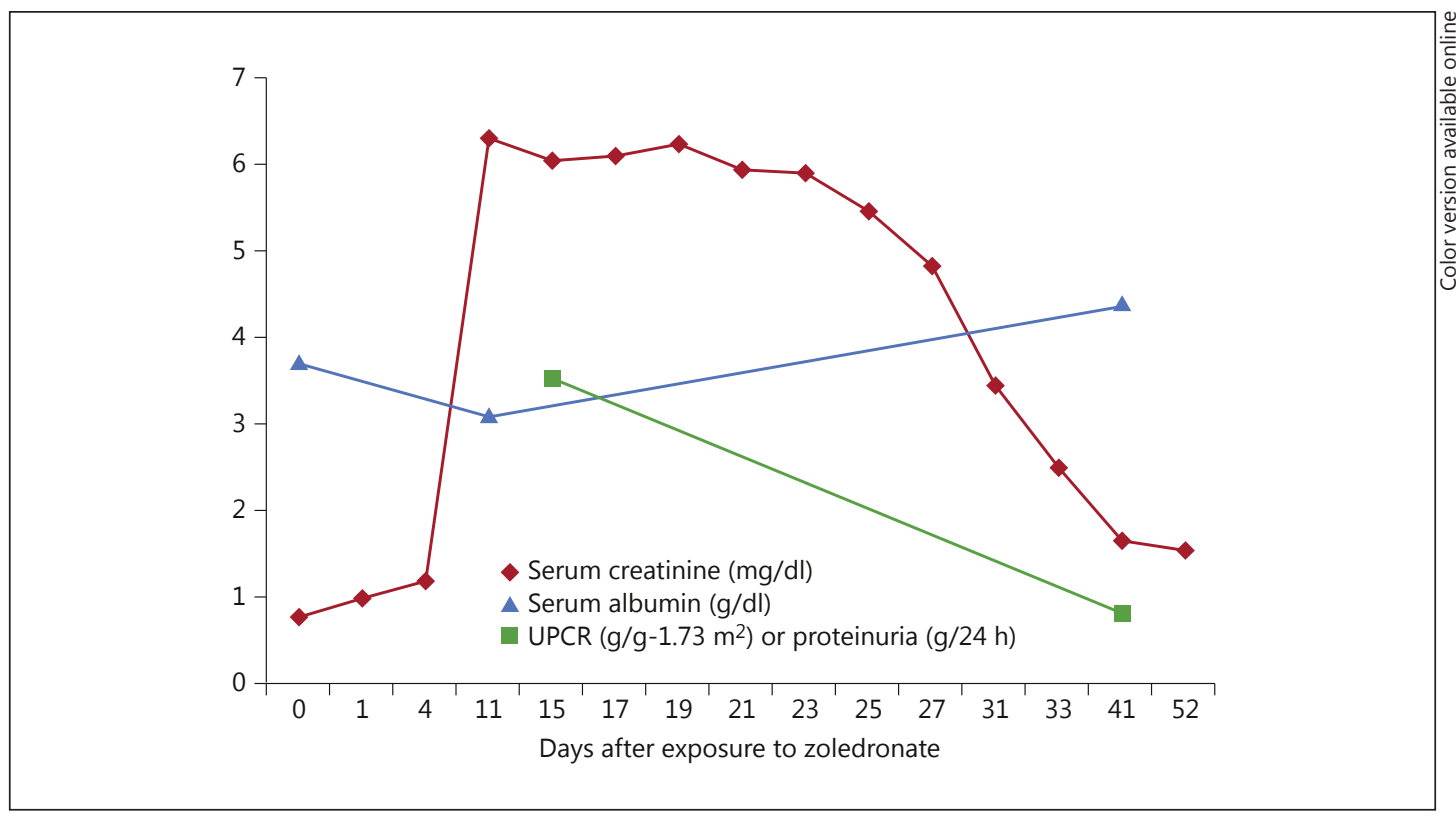

Fig. 3. Clinical course of the renal disease in relation to serum creatinine and proteinuria. Day 0 denotes the exposure to zoledronate. Creatinine is expressed as milligrams or grams to allow the use of a single scale for the y-axis. To convert serum creatinine to $\mu \mathrm{mol} / \mathrm{l}$, multiply values by 88.4. Proteinuria is expressed as $\mathrm{g} / 24 \mathrm{~h}$ when a 24 -hour urine sample was collected and as $\mathrm{g} / \mathrm{g}-1.73 \mathrm{~m}^{2}$ when a spot urine protein-to-creatinine ratio (UPCR) was measured.

\section{Discussion}

Our report describes the case of a young female with breast cancer who developed collapsing FSGS presenting as AKI and nephrotic-range proteinuria after a single dose of zoledronate. The collapsing variant of FSGS is most commonly seen in persons of African descent and can present as idiopathic or secondary forms $[13,14]$. The secondary variant occurs in association with viral infections (HIV, parvovirus and cytomegalovirus), autoimmune diseases (Still's disease and systemic lupus erythematosus) and medications (interferon and bisphosphonates) [13-15].

Based on their molecular structure, bisphosphonates are classified as simple or nitrogencontaining bisphosphonates. Examples of simple bisphosphonates are clodronate, tiludronate and etidronate. No cases of proteinuric renal disease have been reported with this class of bisphosphonates. Nitrogen-containing bisphosphonates include pamidronate, alendronate, zoledronate, minodronate, ibandronate and residronate. Bisphosphonates have a high affinity for calcium ions and are internalized by bone-resorbing osteoclasts prior to inhibiting their function [5]. The nitrogen-containing bisphosphonates act by inhibiting farnesyl diphosphate synthase (FPPS), an enzyme of the mevalonate pathway [16], which is important in creating the lipophilic prenyl groups that are added to some cell membrane-associated proteins during posttranslational modification for normal osteoclast function [16].

The mechanism of how bisphosphonates may induce podocyte dedifferentiation and hyperplasia, the defining characteristics of collapsing FSGS, is unclear. It is interesting to speculate that this may result from podocyte FPPS inhibition. Nitrogen-containing bisphosphonates have been shown to inhibit FPPS in many cell types other than osteoclasts, with the in vivo susceptibility of a cell type likely depending on its ability to internalize sufficient drug. This may explain the typical disease course initially described with pamidronate-induced 
Neyra et al.: Collapsing Focal Segmental Glomerulosclerosis Resulting from a Single Dose of Zoledronate

collapsing FSGS in which glomerulopathy occurred only after multiple doses and prolonged exposure to pamidronate $[9,11,17]$. A gradual accumulation of bisphosphonate in the podocyte may occur prior to effective FPPS inhibition. Once FPPS is inhibited in the podocyte, prenylation of small guanosine-5'-triphosphatases (GTPases) such as those of the Ras, Rho and Rab families may be reduced as has been described in osteoclasts [18]. These signaling proteins regulate a variety of cell processes, including arrangement of the cytoskeleton. Analogous to hereditary forms of FSGS, a proteinuric phenotype may also result from genetic defects in actin regulatory proteins such as $\alpha$ ACTN-4 [19], INF2 [20] or Arhgap24 [21]. The existence of human cases of nephrotic syndrome caused by mutations in proteins just downstream of FPPS in the biosynthesis of coenzyme Q10 lends plausibility to this proposed disease pathway [22].

In addition to affecting protein prenylation, inhibition of FPPS also leads to the accumulation of the upstream substrate isopentenyl diphosphate which reacts with adenosine- $5^{\prime}$ triphosphate (ATP) to form a novel ATP analog (ApppI). ApppI can inhibit mitochondrial adenine nucleotide translocase and induce cell apoptosis [23]. Such an effect may offer an explanation for the mitochondrial proliferation, vacuolization and loss of cristae that has been observed in podocytes in a case of bisphosphonate-induced proteinuria [24]. Additional disease pathways may also be active. Pamidronate, unlike other nitrogen-containing bisphosphonates, maintains antiresorptive effects on osteoclasts even when prenyl groups are added to cell cultures to circumvent the block in FPPS [25]. These alternative molecular targets other than FPPS may explain why pamidronate is the bisphosphonate most often implicated to cause collapsing FSGS.

Our patient did have a history of mild proteinuria attributed to prior therapy with bevacizumab, which is a known cause of proteinuria mediated through vascular endothelial growth factor inhibition [26]. Since there is some evidence that bisphosphonates can also reduce vascular endothelial growth factor levels [27], this may represent a different mechanism by which bisphosphonates may induce podocytopathy. The prior bevacizumab exposure may have put the patient at risk for the development of collapsing FSGS; however, it had occurred 4 years earlier, and the patient had only trace proteinuria on dipstick urinalysis just prior to receiving zoledronate.

Proteinuric renal disease from bisphosphonates other than pamidronate is quite rare. Cases of alendronate-induced proteinuria and collapsing glomerulopathy have been reported less commonly in the literature [28]. While zoledronate is a clear cause of acute tubular injury [8], it is not a known precipitant of collapsing FSGS. To date, there are only 2 cases in the literature in which zoledronate was reported to be associated with collapsing FSGS. In the case by Bodmer et al. [10], a patient developed AKI and proteinuria after the 9th dose of zoledronate. The total duration of exposure was 16 months and there was no renal recovery. Clearly, the chance occurrence of idiopathic FSGS independent of zoledronate exposure cannot be ruled out. Gokden et al. [12] have reported the case of a patient who was on zoledronate for 1 year prior to developing collapsing FSGS. In addition to the possibility of idiopathic disease in this case, there were other possible confounders including the concomitant use of interferon and the prior use of pamidronate. This patient had a partial renal recovery after discontinuation of zoledronate and treatment with steroids.

The present case is unique in multiple respects: the disease occurred immediately after zoledronate exposure and spontaneously remitted with no intervention other than withdrawal of the offending drug. Both make the case for a causative role of zoledronate incontrovertible. In addition, previously reported cases of bisphosphonate-induced FSGS have described a prolonged exposure prior to the onset of disease. In contrast, our patient developed severe acute renal manifestations less than 1 week after her one-time exposure to zoledronate. Lastly, unlike most of the other reported cases of bisphosphonate-induced collapsing 
Neyra et al.: Collapsing Focal Segmental Glomerulosclerosis Resulting from a Single Dose of Zoledronate

FSGS, renal function and proteinuria improved substantially without the introduction of immunosuppression, possibly as a result of limited offending drug exposure.

This case expands the list of known causes of collapsing FSGS to include zoledronate. Periodic monitoring for changes in renal function and new-onset proteinuria is advised when a patient is started on zoledronate. If collapsing FSGS does occur, there may be a chance of partial renal recovery with early discovery and discontinuation of the medication.

\section{Disclosure Statement}

The authors declare that they have no relevant financial interests.

\section{References}

1 Liberman UA, Weiss SR, Bröll J, et al: Effect of oral alendronate on bone mineral density and the incidence of fractures in postmenopausal osteoporosis. The Alendronate Phase III Osteoporosis Treatment Study Group. N Engl J Med 1995;333:1437-1443.

2 Major PP, Coleman RE: Zoledronic acid in the treatment of hypercalcemia of malignancy: results of the international clinical development program. Semin Oncol 2001;28(2 suppl 6):17-24.

-3 Hillner BE, Ingle JN, Chlebowski RT, et al: American Society of Clinical Oncology 2003 update on the role of bisphosphonates and bone health issues in women with breast cancer. J Clin Oncol 2003;21:4042-4057.

4 Hughes DE, Wright KR, Uy HL, et al: Bisphosphonates promote apoptosis in murine osteoclasts in vitro and in vivo. J Bone Miner Res 1995;10:1478-1487.

5 Rogers MJ, Gordon S, Benford HL, et al: Cellular and molecular mechanisms of action of bisphosphonates. Cancer 2000;88(12 suppl):2961-2978.

6 Bounameaux HM, Schifferli J, Montani JP, et al: Renal failure associated with intravenous diphosphonates. Lancet $1983 ; \mathrm{i}: 471$.

7 Zazgornik J, Grafinger P, Biesenbach G, et al: Acute renal failure and alendronate. Nephrol Dial Transplant 1997;12:2797-2798.

-8 Markowitz GS, Fine PL, Stack JI, et al: Toxic acute tubular necrosis following treatment with zoledronate (Zometa). Kidney Int 2003;64:281-289.

-9 Markowitz GS, Appel GB, Fine PL, et al: Collapsing focal segmental glomerulosclerosis following treatment with high-dose pamidronate. J Am Soc Nephrol 2001;12:1164-1172.

10 Bodmer M, Amico P, Mihatsch MJ, et al: Focal segmental glomerulosclerosis associated with long-term treatment with zoledronate in a myeloma patient. Nephrol Dial Transplant 2007;22:2366-2370.

11 Markowitz GS, Fine PL, D’Agati VD: Nephrotic syndrome after treatment with pamidronate. Am J Kidney Dis 2002;39:1118-1122.

12 Gokden N, Zangari M, Elici F, et al: Potential effect of zoledronate therapy in heavy proteinuria. Clin Nephrol 2007;67:263-265.

13 Valeri A, Barisoni L, Appel GB, et al: Idiopathic collapsing focal segmental glomerulosclerosis: a clinicopathologic study. Kidney Int 1996;50:1734-1746.

14 Korbet SM, Genchi RM, Borok RZ, et al: The racial prevalence of glomerular lesions in nephrotic adults. Am J Kidney Dis 1996;27:647-651.

15 Moudgil A, Nast CC, Bagga A, et al: Association of parvovirus B19 infection with idiopathic collapsing glomerulopathy. Kidney Int 2001;59:2126-2133.

16 Roelofs AJ, Thompson K, Gordon S, et al: Molecular mechanisms of action of bisphosphonates: current status. Clin Cancer Res 2006;12(20 Pt 2):6222s-6230s.

$\checkmark 17$ Barri YM, Munshi NC, Sukumalchantra S, et al: Podocyte injury associated glomerulopathies induced by pamidronate. Kidney Int 2004;65:634-641.

-18 Luckman SP, Hughes DE, Coxon FP, et al: Nitrogen-containing bisphosphonates inhibit the mevalonate pathway and prevent post-translational prenylation of GTP-binding proteins, including Ras. J Bone Miner Res 1998;13:581-589.

19 Kaplan JM, Kim SH, North KN, et al: Mutations in ACTN4, encoding alpha-actinin-4, cause familial focal segmental glomerulosclerosis. Nat Genet 2000;24:251-256.

20 Brown EJ, Schlondorff JS, Becker DJ, et al: Mutations in the formin gene INF2 cause focal segmental glomerulosclerosis. Nat Genet 2010;42:72-76.

21 Akilesh S, Suleiman H, Yu H, et al: Arhgap24 inactivates Rac1 in mouse podocytes, and a mutant form is associated with familial focal segmental glomerulosclerosis. J Clin Invest 2011;121:4127-4137. 
22 Lopez LC, Schuelke M, Quinzii CM, et al: Leigh syndrome with nephropathy and CoQ10 deficiency due to decaprenyl diphosphate synthase subunit 2 (PDSS2) mutations. Am J Hum Genet 2006;79:1125-1129.

23 Monkkonen H, Auriola S, Lehenkari P, et al: A new endogenous ATP analog (ApppI) inhibits the mitochondrial adenine nucleotide translocase (ANT) and is responsible for the apoptosis induced by nitrogen-containing bisphosphonates. Br J Pharmacol 2006;147:437-445.

-24 Sauter M, Julg B, Porubsky S, et al: Nephrotic-range proteinuria following pamidronate therapy in a patient with metastatic breast cancer: mitochondrial toxicity as a pathogenetic concept? Am J Kidney Dis 2006;47: 1075-1080.

25 van Beek ER, Cohen LH, Leroy IM, et al: Differentiating the mechanisms of antiresorptive action of nitrogen containing bisphosphonates. Bone 2003;33:805-811.

26 Izzedine H, Massard C, Spano JP, et al: VEGF signalling inhibition-induced proteinuria: mechanisms, significance and management. Eur J Cancer 2010;46:439-448.

27 Santini D, Vincenzi B, Dicuonzo G, et al: Zoledronic acid induces significant and long-lasting modifications of circulating angiogenic factors in cancer patients. Clin Cancer Res 2003;9:2893-2897.

28 Pascual J, Torrealba J, Myers J, et al: Collapsing focal segmental glomerulosclerosis in a liver transplant recipient on alendronate. Osteoporos Int 2007;18:1435-1438. 